\title{
Dynamic Cluster Head Selection Method using LEACH Algorithm for Wireless Sensor Network
}

\author{
Prof. S. U. Patil ${ }^{1}$, Saste. G. S $^{2}$, Ejagar. G. M $^{3}$, Raskar. S. S $^{4}$, Dhumal P.R. ${ }^{5}$ \\ Assistant Prof, Computer science, COEP, Phaltan, India ${ }^{1}$ \\ Student, Computer Science, COEP, Phaltan, India ${ }^{2,3,4,5}$
}

\begin{abstract}
WSNs are used research area due to their several application domains. The presentation of WSNs depends on the topology of sensors and their ability to adapt to changes in the network. Sensor nodes are often resource constrained by their limited power, communication distance capacity is low, and restricted sensing capability. Therefore, they need to co-operate with each other to achieve a specific task. [1] [3] Thus, clustering enables sensor nodes to communicate through the cluster head node for constant communication process. In this paper, we introduce a dynamic cluster head election technique. Every node in the cluster calculates its residual energy value to determine its candidacy to become the Cluster Head Node (CHN). [1] With this mechanism, each sensor node estimates its residual energy level to other nodes in the same cluster. Depending on the residual energy level the sensor node acts as the second cluster head. Evaluation of the dynamic CHN election mechanism is shown using network simulator-2 (ns2) . The simulation results show that the proposed approach prolongs the network lifetime and balancing the energy consumption model among the nodes of the cluster. [2]
\end{abstract}

Keywords: Cluster head, Coverage, Redundant Node, Energy, Lifetime.

\section{INTRODUCTION}

Wireless sensor networks involvement limited depletion among self-powered sensor nodes within communication bandwidth and energy constraints. WSN is clusters.[2]

based on data-centric wireless network that does not need focusing on sender and receiver. Unlike, traditional wired network, mobile wireless network and ad hoc network care extra about the sender and receiver.[2] Therefore, a general IP based mechanism and multi-hop routing scheme for mobile ad hoc network is not suitable for WSNs. The hierarchical routing protocol is one kind of typical network protocol for WSNs to handle the faults of the flat traditional cluster based routing scheme. Thus it spreads the network lifetime as well as guarantees better connectivity of whole network. [1]

Energy feasting is one of the serious problems in WSNs that creates challenges for academic and industrial sectors. Therefore, energy handling is one of the key skills to spreads the network lifetime.[2] There is a quadratic increase in energy ingesting as the distance among sensors increases. Thus, the distance should be kept under thought while designing the WSNs to minimize energy consumption and prolong network lifetime.[3]

Scalability is the second major threat in WSNs where thousands of sensor nodes are deployed in confident applications. In WSN's these matters are addressed at the cluster level by using different cluster-based architectures. [1]Leach algorithm introduced cluster-based architecture where the cluster head static and its energy is consumed rapidly due to the extra charge of data aggregation. The cluster head selection and formation scheme consumes been introduced in for handling the unbalanced energy

The cluster head in decides to choose a timeframe on based on the energy consumed in the recent round but it is probability based scheme. [6] The dynamic clustering scheme for WSNs is introduced in for focusing head node selection, cluster numbering and cluster reorganization for reducing energy consumption definite on signal strength and distance between the sink and the cluster head. [5] To control scalability, a clustering algorithm is planned for selection of the cluster head node based on the received signal strength of node and the distance between the cluster head node and the sink node.[8]

Another dynamic clustering algorithm is presented in using genetic algorithm. The algorithm considers different parameters to recover the network lifetime but most focusing on distance while selecting cluster head node. A cluster header selection scheme is proposed with different cluster creation mechanism in.[6] The node is selected as cluster head node on the basis of its comparative energy consumption in the recent round. This scheme tries to increase network lifetime and suggestions a balanced energy consumption configuration among the nodes.[5]

\section{LITERATURE SURVEY}

In LEACH (Low-Energy Adaptive Clustering Hierarchy) protocol the data is guided to the clusterheads who in efforts forward the same to the basin node after collecting. 


\section{ISO 3297:2007 Certified}

Vol. 5, Issue 3, March 2017

The rounds in theLEACH operation are divided into two namely - a set-up phase and a steady-state phase.[4]

Organization of the clusters is comprehensive in the set-up phase and the data is guided to their clusterheads in the steady-state phase. Cluster Heads recurrence after every Prounds, where $\mathrm{P}$ is thepercentage of the cluster heads.[2] Therefore each round has $1 / \mathrm{P}$ probability of attractive acluster head in each round3, 4. A node that is not a cluster head selects a new cluster head thatis closest to it at the end of each round and joints that cluster head. Head than creates a Schedule for each node to communicate the data.

Power Efficient Gathering in Sensor Information Systems (PEGASIS) PEGASIS is a chain-based protocol and an development over LEACH protocol. In PEGASIS, each node communicates with its instantneighbor and finally transmits to the base station turnby turn4, 7. Greedy algorithm or the phenomena of sending the chains by the sink nodes to thesensor nodes can be useful in organizing the sensors into chain. [5] Only one node is permissible toaggregate the data and send it to sink. However this protocol needs a broader knowledge of the

complete topology of the network whereby increasing the complexity of the protocol. Sincethere is only a secure data path followed by every node, so in case of node failures determining anew path is often difficult .This protocol supports in conserving energy but it does not focus muchon the quality of service issue.

\section{PROBLEM DEFINITION}

Problem Statement - Clustering data with consecutive constraints is a polynomial time solvable variant of the clustering problem. As the selection of clusters is the tedious job. Energy consumption is one of the serious problems in WSNs that creates tasks for academic and industrial sectors. [1] Therefore, energy handling is one of the key skills to spread the network lifetime. There is a quadratic rise in energy consumption as the distance among sensors increases. Thus, the distance should be reserved under consideration while designing the WSNs to minimize energy consumption and prolong network lifetime. [3]

\section{SOLVING APPROACH}

The paper introduced a package implementing a dynamic programming approach that finds the exact finest of the problem. The algorithm represents an allowance of the one-dimensional dynamic programming strategy of $\mathrm{CHN}$ election to multiple dimensional spaces which has been an open problem in the paper of Wang and Song (2011). The package provisions both cases when the exact number of clusters is given and when the number of clusters is not known in advance. It can also be used to evaluate approximation algorithms for clustering with consecutive constraints due to its optimality. The runtime evaluations show that how fast the algorithm can solve problems with diverse sizes and parameters.

Process of cluster Heads optimize selection

The enhanced cluster heads selection process is shown in Figure 1. Definite steps are showed below:

1. Initiating the network. The base station can get location of all the sensor nodes in checking area (ID) and the residual energy of the nodes.

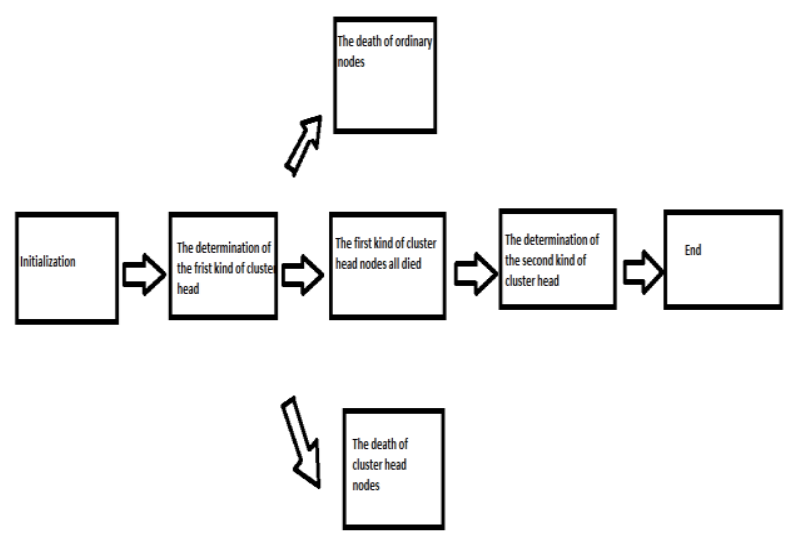

Fig: The process of cluster heads optimized selection

2. The monitoring area is divided into some clusters by Verona diagram, and the insight probabilistic model is proposed. Select network dismissed nodes by the attenuation probabilistic algorithm and these nodes are taken as the first kind of hibernation cluster head node.

3 . The death of a current cluster head node makes another terminated node active to be the cluster head. If the death node is a current common node, another redundant node ends latency to be an ordinary node.

4. The survival time estimation algorithm can be used to estimate the network typical residual energy, if the first kind of cluster head nodes all died. Select the second class of cluster head nodes created on the ratio of the residual energy and the average energy of the network nodes.

The impact of redundant nodes and network residual energy on network is larger in the process of practical research, which makes the redundant nodes and the average residual energy mainlyexamined in this paper.

\section{CONCLUSION}

The predictable output, Dynamic cluster head node selection (DCHNS) model over WSNs to prolong the network lifetime.[2] We have displayed dynamic election process of cluster head node. The cluster head node is elected on the source of residual energy of sensor nodes. The residual energy is calculated after accomplishment the event monitoring process using the mathematical model. In our scheme, the nodes can switch to their special of cluster even with increased power loads. To establish the 
strength of DCHNE, we have used ns2 simulator that illustrates its performance efficiency. Two types of scenarios are used by algorithm which is characterized by the amount of activity supposed in the environments. On basis of simulation results and the mathematical model, we believe that the proposed scheme significantly extends the network lifetime as compared with other schemes.[3]

\section{REFERENCES}

1. Z. H. Li et al, "Efficient and dynamic clustering scheme for heterogeneous multi-level wireless sensornetwork". International Journal of science Direct on ACTA AUTOMATICA Sanica, vol. 39, no.4, 2013,pp.454-460.

2. Abbasi A A, Younis M, Razaque and K.M. Elleithy, "Least Distance Smart neighboring Search (LDSNS) over Wireless Sensor Networks",IEEE European Modelling Symposium EMS2013, Manchester, 20 - 22 November 2013

3. Nivedita Rajani, "Energy Efficient, Reliable Routing Protocol for Wireless Sensor",International Journal of Computer Science and Technology, Vol 1, Issue 4, June 2008, pp 6-14.

4. W. Rabiner et al., "Energy Efficient, Reliable Routing Protocol for Wireless Sensor",33rd Hawaii International Conference on System Sciences, 2009, pp 63-71.

5. M. Hossein et al., "RDAG: A Structure-free Real time Data Aggregation Protocol for Wireless Sensor Networks"IEEE International Conference on Embedded and Real-Time Computing Systems andApplications, July 2010, China, pp 94- 102 .

6. J. Zhang et al."Effective data aggregation supported by dynamic routing in wireless sensornetworks"IEEE International Conference on Communications (ICC'10), pp 55-64, May 2010.

7. J.Baek et al., "Dynamic cluster header selection with Self- incentive for wireless sensor networks",In proceedings of IEEE Sarnoff Symposium on SARNOFF, Princeton, NJ, pp.1-5, 2009.

8. Z.K.Liu, and Z. Liu, “A dynamic clustering protocol for wireless sensor networks",in proceedings of IEEE international conference on computational and information sciences (ICCIS), Chengdu, China, pp.777-779, 2011. 